\title{
Intracameral Air and Fibrin Glue for the Management of Late Bleb Leak following Glaucoma Surgery
}

\author{
Mohammed R. Hayat ${ }^{a}$ Fatema S. Mollah ${ }^{a}$ Kamal Kishore ${ }^{a, b}$ \\ aUniversity of Illinois College of Medicine, Peoria Campus, Peoria, IL, USA; \\ bIllinois Retina and Eye Associates, Peoria, IL, USA
}

\section{Keywords}

Intracameral air · Fibrin glue · Glaucoma · Bleb leak · Bleb-associated endophthalmitis

\begin{abstract}
Background and Objectives: To report a case of delayed-onset bleb-associated endophthalmitis (BAE) with bleb leak successfully managed with pars plana vitrectomy, intravitreal antibiotics, intracameral air, and fibrin glue. Patient and Methods: A 66-year-old pseudophakic female presented with BAE and bleb leak. A 25-gauge pars plana vitrectomy, cultures, and intravitreal antibiotics and steroid injections were performed. The infusion was switched to air filling the anterior chamber and bleb with air. Fibrin glue $\left(\right.$ Tisseel $\left.^{\circledR}\right)$ was applied over the leaking bleb. Results: BAE and bleb leak resolved with return of visual acuity to 20/25 and a functioning bleb with no recurrence of bleb leak after 1 year of follow-up. Conclusion: The combination of intracameral air and fibrin glue may have a role in the management of bleb leaks.
\end{abstract}

(C) 2019 The Author(s)

Published by S. Karger AG, Basel

\section{Introduction}

Late-onset bleb leaks, seen in approximately $25 \%$ of the eyes following trabeculectomy with mitomycin $\mathrm{C}$, can result in serious complications including bleb-associated endophthalmitis (BAE) in some eyes [1]. While mild, asymptomatic bleb leaks may seal spontaneously 
with observation, hypotony, bleb infection, or blurred vision are generally accepted indications for the treatment of bleb leaks $[1,2]$. Various nonsurgical and surgical approaches have been described in the literature to manage bleb leaks [2,3]. Surgical methods such as bleb excision with conjunctival flap advancement or autologous conjunctival flap are more effective than nonsurgical methods but may be technically difficult and result in conjunctival retraction, ptosis, bleb failure, and elevation of intraocular pressure (IOP) $[2,4]$.

Application of fibrin glue for posttrabeculectomy bleb leaks has been reported in the past [5-8]. Asrani and Wilensky [7] in 1996 applied autologous fibrin tissue glue over the surface of a leaking bleb with a success rate of approximately $75 \%$, which was comparable to other nonsurgical treatments in their study. Grewing and Mester [9] injected fibrin sealant subconjunctivally over the scleral flap to achieve temporary tamponade of filtration in two cases of choroidal detachments due to hypotony following trabeculectomy. Choroidal detachments resolved in both cases. However, none of the eyes had bleb leaks. Seligsohn et al. [8] injected fibrin sealant (Tisseel ${ }^{\circledR}$ ) into the bleb in seven eyes, four with bleb leaks and three with hypotony without bleb leak, without much success and concluded that "Tisseel ${ }^{\circledR}$ may not be an effective treatment of leaking blebs or hypotony following trabeculectomy."

Treatment of significant bleb leaks in the setting of BAE poses serious problems. The most common approach consisting of bleb excision, debridement of necrotic tissue, and conjunctival advancement flap to cover the trabeculectomy site can be technically difficult and may result in bleb failure [10].

In this communication, we describe a case of BAE associated with significant bleb leak that was successfully managed with 25-gauge pars plana vitrectomy, intravitreal antibiotics, intracameral air, and off-label application of fibrin glue. The study was performed in accordance with the tenets of the Declaration of Helsinki (2004) and informed consent was obtained from the patient. Approval from an institutional review board was not required for this case report involving a single patient.

\section{Case Report}

A 66-year-old pseudophakic patient with a history of trabeculectomy with adjunctive mitomycin C left eye 6 years ago presented with a 2-day history of pain, redness, and decreased vision. Examination revealed best-corrected visual acuity of 20/200 OS, diffuse conjunctival congestion, purulent material in the bleb, corneal edema, and fibrin and 4+ cells in the anterior chamber (Fig. 1). There was a well-centered posterior chamber intraocular lens with an open capsule. The intraocular pressure (IOP) was zero due to obvious leak from the bleb. Fundus details were not visible due to dense vitritis. The right eye was unremarkable with a visual acuity of $20 / 20$, IOP of $10 \mathrm{~mm} \mathrm{Hg}$, and a cystic bleb superiorly secondary to glaucoma filtration surgery with mitomycin $\mathrm{C}$.

A diagnosis of acute BAE was made. She underwent 25-gauge pars plana vitrectomy on the same day. Obvious leakage from the bleb was observed intraoperatively (see online video, www.karger.com/doi/10.1159/000496383). Undiluted sample of vitreous was obtained by performing vitrectomy under air infusion. Vitreous and aqueous samples were sent for Gram stain and bacterial and fungal cultures. Vitrectomy was completed after turning the infusion to fluid. After completion of vitrectomy, infusion was switched back to air to leave the vitreous cavity partially filled with air and allowing the air to enter the anterior chamber and the bleb. Intravitreal injections of vancomycin $1 \mathrm{mg} / 0.1 \mathrm{~mL}$, ceftazidime $2.25 \mathrm{mg} / 0.1 \mathrm{~mL}$, and dexamethasone $400 \mu \mathrm{g} / 0.1 \mathrm{~mL}$ were administered. Fibrinogen solution followed by thrombin 
solution of a commercially available fibrin glue (Tisseel ${ }^{\circledR}$, Baxter Inc., Deerfield, IL, USA) was applied off-label over the bleb by two separate syringes. After allowing the glue to dry and form a transparent jelly-like film, excess glue was excised with forceps and Vannas scissors. The eye was patched overnight. Postoperatively, she was placed on $1 \%$ prednisolone acetate drops every $2 \mathrm{~h}$ while awake and $0.3 \%$ ciprofloxacin eye drops q.i.d., which were tapered over 2 weeks.

Her endophthalmitis resolved. Cultures from both the anterior chamber and vitreous were negative. The visual acuity improved to 20/25 2 weeks after surgery and has remained at that level through 1-year follow-up. She continues to have a functioning glaucoma filter with IOP of $12 \mathrm{~mm} \mathrm{Hg}$ without any antiglaucoma medications at the 1-year follow-up visit without any episodes of bleb leak (Fig. 2).

\section{Discussion}

Delayed-onset BAE is a serious and potentially sight-threatening complication of glaucoma filtration surgery with a 5-year incidence of about 7.5\% [11]. The most common organisms responsible of BAE include Streptococcus, Staphylococcus epidermidis, Enterococcus, and Haemophilus influenzae [12-14]. Pars plana vitrectomy may be superior to tap-and-inject procedure in the management of BAE [14].

Bleb leak is a common and serious complication of trabeculectomy with antimetabolites, and if significant, can lead to hypotony, shallow or flat anterior chamber, hypotony maculopathy, choroidal effusions, suprachoroidal hemorrhage, blebitis, and BAE [2]. Several nonsurgical and surgical options are available to manage bleb leaks. Nonsurgical methods such as pressure patching, aqueous suppression, soft contact lens, collagen shield, Simmons shell, cyanoacrylate glue, and autologous blood or serum may be effective in some cases [2, 3, 7]. The most common surgical approaches consist of a conjunctival hood flap to cover the existing bleb or bleb excision and conjunctival advancement. In addition, suture closure of the ostium, or scleral or human pericardial patch grafts may be necessary to manage the eyes with excessive flow from the filtration opening $[2,3,15,16]$. However, patch grafts might not be advisable in the setting of endophthalmitis.

Fibrin glue is commonly used off-label for various ophthalmic indications [17]. In the specialty of glaucoma, it has been used for the fixation of the plate of glaucoma drainage device [18], secure the tube of glaucoma drainage device to the sclera, secure the patch graft, conjunctival closure in both glaucoma drainage device surgery and trabeculectomy [19, 20], and recently as an adjunct to sliding conjunctival flap for the surgical repair of bleb leaks [21].

Kajiwara [5] reported successful repair of a leaking conjunctival wound following trabeculectomy with a commercially available fibrin glue. Fibrinogen and thrombin solutions were applied alternately directly over the suture line for a total of three applications. Graham et al. [6] employed autologous fibrin glue to repair early posttrabeculectomy bleb leaks following fornix-based surgery. Although Asrani and Wilensky [7] observed 75\% success rate of autologous fibrin glue in the management of bleb leaks, it was not much different from the other nonsurgical methods. Kajiwara [5], Graham et al. [6], and Asrani and Wilensky [7] applied fibrin glue over the leaking bleb after drying the area as much as possible. Seligsohn et al. [8] injected commercially available fibrin glue (Tisseel ${ }^{\circledR}$ ) into the leaking bleb and noted very limited efficacy. Since fibrin glue requires a dry surface to be effective, application of fibrin glue over a continuously leaking bleb as done by Asrani and Wilensky [7] might not allow a stable fibrin clot to form. We allowed intravitreal air to enter the anterior chamber and fill 
the bleb to seal the leak by high surface tension of the air-aqueous interface. In addition, the air also helps to create a dry surface that makes the glue more effective [17].

Intracameral injection of SF6 gas has been used in the management of hypotony following glaucoma surgery presumably by reducing filtration through the bleb [22]. None of those patients had obvious bleb leaks. To our knowledge, the use of intracameral air or gas has not been reported for the management of bleb leaks. Compared to other surgical approaches described in the literature, intracameral air and fibrin glue may constitute a simple and straightforward technique for the management of bleb leaks.

To conclude, the combination of intracameral air and off-label fibrin glue may work synergistically in the management of bleb leaks and warrants further study.

\section{Statement of Ethics}

All procedures were done in accordance with the tenets of Helsinki Declaration of 1964, as revised in 2013. Approval from an institutional review board was not required for this retrospective study involving a single case report.

\section{Disclosure Statement}

The authors have no financial disclosures to make and no proprietary interests to disclose. There are no conflicts of interest for any of the authors.

\section{References}

1 Anand N, Arora S, Clowes M. Mitomycin C augmented glaucoma surgery: evolution of filtering bleb avascularity, transconjunctival oozing, and leaks. Br J Ophthalmol. 2006 Feb;90(2):175-80.

2 Feldman RM, Altaher G. Management of late-onset bleb leaks. Curr Opin Ophthalmol. 2004 Apr;15(2):151-4.

3 Mandal AK. Management of the late leaking filtration blebs. A report of seven cases and a selective review of the literature. Indian J Ophthalmol. 2001 Dec;49(4):247-54.

4 Senthil S, Basia A, Rao HL, Choudhari N, Dikshit S, Mandal AK, et al. Outcomes of bleb repair for delayed bleb leaks and sweating blebs. J Glaucoma. 2018 Jan;27(1):33-40.

5 Kajiwara K. Repair of a leaking bleb with fibrin glue. Am J Ophthalmol. 1990 May;109(5):599-601.

6 Graham SL, Murray B, Goldberg I. Closure of fornix-based posttrabeculectomy conjunctival wound leaks with autologous fibrin glue. Am J Ophthalmol. 1992 Aug;114(2):221-2.

7 Asrani SG, Wilensky JT. Management of bleb leaks after glaucoma filtering surgery. Use of autologous fibrin tissue glue as an alternative. Ophthalmology. $1996 \mathrm{Feb}$;103(2):294-8.

8 Seligsohn A, Moster MR, Steinmann W, Fontanarosa J. Use of Tisseel fibrin sealant to manage bleb leaks and hypotony: case series. J Glaucoma. 2004 Jun;13(3):227.

9 Grewing R, Mester U. Fibrin sealant in the management of complicated hypotony after trabeculectomy. Ophthalmic Surg Lasers. 1997 Feb;28(2):124-7.

10 Ohtomo K, Mayama C, Ueta T, Nagahara M. Outcomes of late-onset bleb-related endophthalmitis treated with pars plana vitrectomy. J Ophthalmol. 2015;2015:923857.

11 DeBry PW, Perkins TW, Heatley G, Kaufman P, Brumback LC. Incidence of late-onset bleb-related complications following trabeculectomy with mitomycin. Arch Ophthalmol. 2002 Mar;120(3):297-300.

12 Greenfield DS, Suñer IJ, Miller MP, Kangas TA, Palmberg PF, Flynn HW Jr. Endophthalmitis after filtering surgery with mitomycin. Arch Ophthalmol. 1996 Aug;114(8):943-9.

13 Song A, Scott IU, Flynn HW Jr, Budenz DL. Delayed-onset bleb-associated endophthalmitis: clinical features and visual acuity outcomes. Ophthalmology. 2002 May;109(5):985-91.

14 Busbee BG, Recchia FM, Kaiser R, et al. Bleb-associated endophthalmitis: Clinical characteristics and visual outcomes. Ophthalmology 2004;111(8):1495-503; discussion 1503.

15 Kosmin AS, Wishart PK. A full-thickness scleral graft for the surgical management of a late filtration bleb leak. Ophthalmic Surg Lasers. 1997 Jun;28(6):461-8. 


\section{Case Reports in Ophthalmology}

\begin{tabular}{l|l}
\hline Case Rep Ophthalmol 2019;10:47-52 \\
\hline DOI: 10.1159/000496383 & $\begin{array}{l}\text { @ 2019 The Author(s). Published by S. Karger AG, Basel } \\
\text { www.karger.com/cop }\end{array}$ \\
\hline
\end{tabular}

Hayat et al.: Intracameral Air and Fibrin Glue for the Management of Late Bleb Leak following Glaucoma Surgery

16 Tannenbaum DP, Hoffman D, Greaney MJ, Caprioli J. Outcomes of bleb excision and conjunctival advancement for leaking or hypotonous eyes after glaucoma filtering surgery. Br J Ophthalmol. 2004 Jan;88(1):99-103.

17 Panda A, Kumar S, Kumar A, Bansal R, Bhartiya S. Fibrin glue in ophthalmology. Indian J Ophthalmol. 2009 Sep-Oct;57(5):371-9.

18 Harvey JD, Gross RL, McMillan BD. Novel use of fibrin sealant for scleral suture free placement of a glaucoma drainage device in advanced scleral thinning. Am J Ophthalmol Case Rep. 2017 Oct;9:7-9.

19 O'Sullivan F, Dalton R, Rostron CK. Fibrin glue: an alternative method of wound closure in glaucoma surgery. J Glaucoma. 1996 Dec;5(6):367-70.

20 Kahook MY, Noecker RJ. Fibrin glue-assisted glaucoma drainage device surgery. Br J Ophthalmol. 2006 Dec;90(12):1486-9.

21 Lee GA, Holcombe DJ. Surgical revision of dysfunctional filtration blebs with bleb preservation, sliding conjunctival flap and fibrin glue. Eye (Lond). 2010 Jun;24(6):947-53.

22 Gotzaridis SV, Portaliou DM, Mela VA, Xanthopoulou P, Kymionis GD. Anterior chamber sulfur hexafluoride (SF6) injection for the management of postsurgery hypotony in glaucoma patients. J Glaucoma. 2018 May;27(5):476-9.

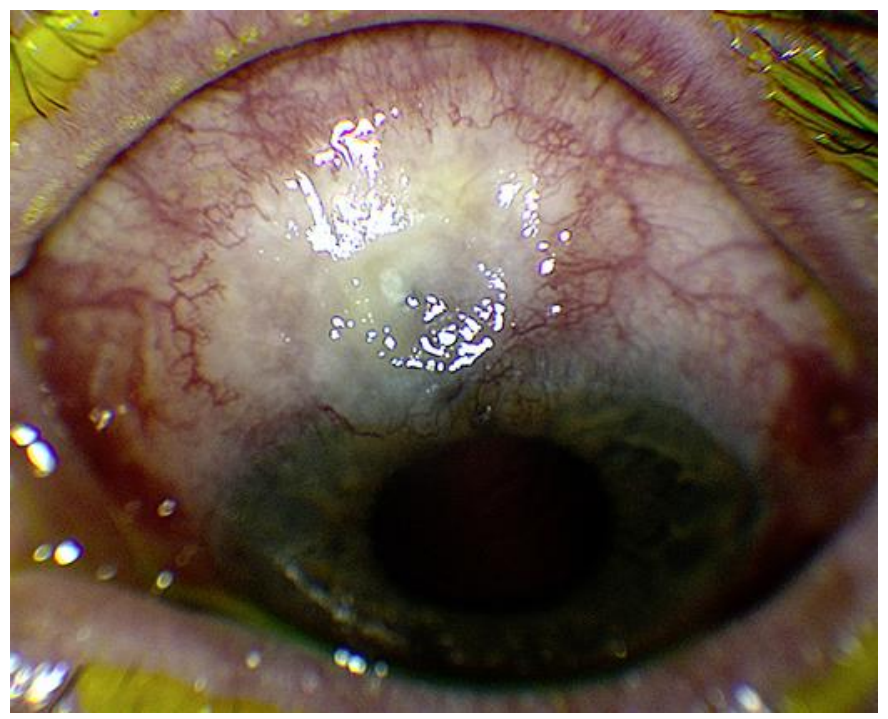

Fig. 1. Anterior segment photograph of the left eye at presentation showing conjunctival congestion and avascular bleb with central ulceration. 


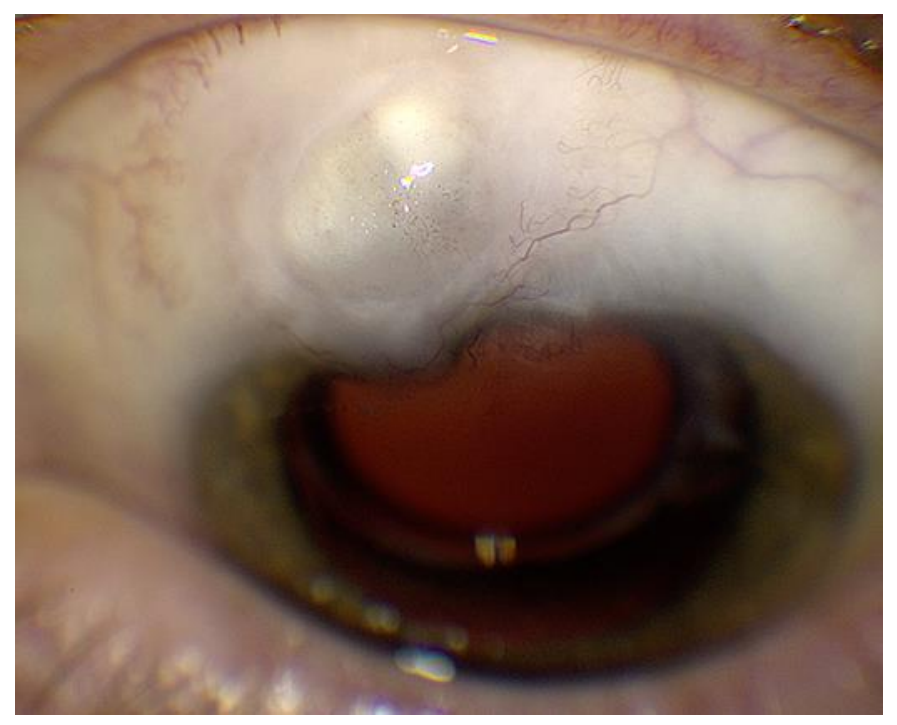

Fig. 2. Anterior segment photograph of the left eye 1 year after pars plana vitrectomy, intravitreal antibiotics, and repair of bleb with intracameral air and fibrin glue. 